\title{
Systematic review of antiepileptic drugs' safety and effectiveness in feline epilepsy
}

\author{
Marios Charalambous ${ }^{1 *} \mathbb{D}$, Akos Pakozdy ${ }^{2}$, Sofie F. M. Bhatti ${ }^{1}$ and Holger A. Volk ${ }^{3}$
}

\begin{abstract}
Background: Understanding the efficacy and safety profile of antiepileptic drugs (AEDs) in feline epilepsy is a crucial consideration for managing this important brain disease. However, there is a lack of information about the treatment of feline epilepsy and therefore a systematic review was constructed to assess current evidence for the AEDs' efficacy and tolerability in cats. The methods and materials of our former systematic reviews in canine epilepsy were mostly mirrored for the current systematic review in cats. Databases of PubMed, CAB Direct and Google scholar were searched to detect peer-reviewed studies reporting efficacy and/or adverse effects of AEDs in cats. The studies were assessed with regards to their quality of evidence, i.e. study design, study population, diagnostic criteria and overall risk of bias and the outcome measures reported, i.e. prevalence and $95 \%$ confidence interval of the successful and affected population in each study and in total.

Results: Forty studies describing clinical outcomes of AEDs' efficacy and safety were included. Only two studies were classified as "blinded randomised controlled trials". The majority of the studies offered high overall risk of bias and described low feline populations with unclear diagnostic criteria and short treatment or follow-up periods. Individual AED assessments of efficacy and safety profile showed that phenobarbital might currently be considered as the first choice AED followed by levetiracetam and imepitoin. Only imepitoin's safety profile was supported by strong level of evidence. Imepitoin's efficacy as well as remaining AEDs' efficacy and safety profile were supported by weak level of evidence.

Conclusions: This systematic review reflects an evidence-based assessment of the published data on the AEDs' efficacy and safety for feline epilepsy. Currently, phenobarbital is likely to be the first-line for feline epileptic patients followed by levetiracetam and imepitoin. It is essential that clinicians evaluate both AEDs' effectiveness and tolerability before tailoring AED to the individual patient. Further studies in feline epilepsy treatment are by far crucial in order to establish definite guidelines for AEDs' efficacy and safety.
\end{abstract}

Keywords: comprehensive review, epilepsy, feline, antiepileptic drugs, efficacy, adverse effects

\section{Background}

There is a paucity of literature about feline epilepsy compared to canine epilepsy, however epileptic seizures are a common neurological manifestation in cats with an estimated prevalence in a referral hospital population of $0.5-3.5 \%[1,2]$. Idiopathic epilepsy (IE) has not been studied in cats as thoroughly as in dogs but between $21-59 \%$ of cats presenting with recurrent seizures can be diagnosed with IE [3-5].

\footnotetext{
* Correspondence: marios.charalambous@ugent.be

${ }^{1}$ Small Animal Department, Faculty of Veterinary Medicine, Ghent University, Merelbeke, Belgium

Full list of author information is available at the end of the article
}

Treatment between canine and feline epileptic patients is rather different mainly due to the divergent safety profile between the two species [6,7] but it is acceptable that in both species the effectiveness of an AED may outweigh its adverse effects, and therefore both the efficacy and safety profile should be considered before choosing the most appropriate $\operatorname{AED}(\mathrm{s})$ for patients [8].

Systematic reviews are powerful and objective tools to evaluate AEDs' efficacy as well as severity and incidence of AEDs' safety profile described in literature [9-12]. Guidelines with regards to AEDs' efficacy and safety have been established in canine epilepsy $[13,14]$ based on previous systematic reviews and meta-analysis $[8,15]$. However, such guidelines lack in feline epilepsy. The 
goal of this systematic review was to summarize and assess the results of the current studies regarding AEDs' efficacy and safety in cats and provide evidence on the treatment of feline epilepsy.

\section{Methods}

The methodology followed in this study was based on previous similar studies published by the authors $[8,15]$.

\section{Search strategy}

Studies evaluating or describing the effectiveness and safety of an AED in cats were searched. As it was described in our previous systematic reviews $[8,15]$, studies were assessed based on four inclusion criteria adapted for feline patients, i.e. type of study (any peer-reviewed study), case definition (cats that were investigated and diagnosed with confirmed or presumed idiopathic epilepsy), treatment (cats treated with antiepileptic drugs only) and outcome (description of efficacy or safety outcomes after treatment). Final electronic searches were carried out on 21 June 2017 with no date or language restrictions and the same databases were searched with the same search strategies as it was described before $[8,15]$, i.e. Pub Med, CAB Abstracts, Google Scholar and searching of articles, proceedings and textbooks to identify reference lists of published papers and proceedings of relevant scientific conferences such as the annual American College of Veterinary Internal Medicine forum and the European College of Veterinary Neurology Symposium. The search terms used for the electronic databases were also the same as in our two previous systematic reviews on canine $[8,15]$ but adapted for feline subjects, i.e. the terms 'dog' and 'canine' were replaced by the terms 'cat' and 'feline', respectively.

\section{Study Selection}

The same two-stage screening process that was recruited in our previous systematic reviews $[8,15]$ was used; in summary, at stage 1 , papers' titles and abstracts were only evaluated and at stage 2, full-length papers were evaluated based on the inclusion criteria 2,3 and 4 to exclude the ones irrelevant to our outcomes.

\section{Assessment of quality of evidence}

As in our previous systematic reviews [8, 15], studies were categorised based on their study design, i.e. "blinded randomised clinical trials" (bRCTs), "blinded randomised experimental laboratory animal studies" (bRELAS), "non-blinded RCTs" (nbRCTs), "non-blinded randomised ELAS" (nbRELAS), "non-randomised clinical trials" (NRCTs), "non-randomised ELAS" (NRELAS), "uncontrolled clinical trials" (UCTs), "uncontrolled ELAS" (UELAS), "case series and case reports [16, 17]". In addition, the same three-part system of evidence quality evaluation to characterize weaknesses and strengths of each study within each group was used as it was described in our previous systematic reviews $[8,15]$, i.e. "(a) study group sizes, (b) subject enrolment quality and (c) overall risk of bias based on Cochrane [18] and Syrcle's [19] 'risk of bias' assessment tool in order to provide an indicator of confidence associated with the findings of each study". All in all, bRCTs or bRELAS with large population, clear and thorough diagnostic criteria and low overall risk of bias were considered as studies with the highest quality of evidence. Lastly, the treatment or follow-up period was reported for each study and was considered short or long if it was less or more than 6 months, respectively.

\section{Study group sizes}

The same system that was reported in our previous systematic reviews $[8,15]$ was used to evaluate the population size in each study, i.e. "(a) $>50$ subjects per group ('good' number), (b) 20-50 subjects ('moderate' number), (c) 10-19 subjects ('small' number) and (d) $<10$ subjects ('very small' number)".

\section{Assessment of subject enrolment quality}

Information with regards to the investigations used for the diagnosis of IE were reviewed to assess the quality of subject definition in each study as 'well characterized', 'fairly characterized, 'poorly characterized' or 'unclear.' As far as the ELAS are concerned, that included investigations for describing non-epileptic healthy animals, two categories were described, i.e. 'clearly characterized' and 'unclear'. The systems used were adapted for feline patients from our previous systematic reviews $[8,15]$. The main difference is that for the "well characterized" category, tests for infectious diseases (including feline leukemia virus, feline immunodeficiency virus, feline infectious peritonitis, toxoplasmosis) were also included but these were desirable but not mandatory.

\section{Assessment of overall risk of bias and level of the studies' evidence}

The same methodology followed in our previous systematic review was used [8], i.e. "the studies were assessed based on the 'risk of bias' components and were categorised as presenting 'high', 'low' or 'unclear' risk for each component. The components were the random sequence generation, allocation concealment, blinding of participants, personnel and outcome assessment, completeness of outcome data, selective reporting of outcomes, random housing and baseline characteristics of cats (only for ELAS) and other sources of bias". 


\section{Assessment of outcome measures}

The outcome measure was the assessment of the effectiveness and tolerability of $\operatorname{AED}(\mathrm{s})$ administered in cats.

\section{AEDs' effectiveness}

The assessment of the effectiveness was performed based on our previous canine systematic review [15]. In addition, the 95\% confidence interval (CI) of the successful population in each study was also calculated and assessed as previously [15]; in summary, in each study, the cats with $\geq 50 \%$ reduction in seizure frequency were considered as successful cases and if the 95\% CI of successfully treated cases was within ranges greater than $50 \%$, then it was assumed that the majority of the study population was successfully treated.

\section{AEDs' safety}

The adverse effects were categorised by organ system, i.e. neurological, gastro-intestinal, etc. and types, i.e. type I (dose-dependent) and type II (idiosyncratic or doseindependent). The assessment of the safety was performed based on our previous systematic review [8]; in summary, the prevalence of the affected population in each study (i.e. number of cats that experienced adverse effects divided by the total size of the study population) and proportion of specific adverse effects for each individual AED (i.e. number of studies that reported a specific adverse effect divided by the total number of the studies for this AED) were evaluated. A further outcome measure was also added which was the proportion of specific adverse effects for each AED based on the total affected population (i.e. number of subjects that developed a specific adverse effect divided by the total population size from all the studies). In addition, the 95\% CI of the affected population in each study was also calculated and assessed as previously [8]. If the 95\% CI of the affected cases was within ranges greater than $50 \%$, then it was considered that the majority of the study population showed adverse effects.

\section{Results}

\section{Description of studies}

By 21 June 2017, a total number of 684 unique citations were found; 676 articles and eight major conference abstracts from manual and electronic searches. One hundred seventy six items fulfilled stage 1 screening criteria, of which, 40 final studies (published between 1973 and 2017) also fulfilled stage 2 selection criteria and were therefore chosen for thorough evaluation. The process is also shown via a flow diagram (Additional file 1). One study included three different trials [20] and another study included both a trial and a retrospective part [21]. The included studies represented 2/40 (5\%) bRELAS, $1 / 40$ (2.5\%) nbRCT, 2/40 (5\%) nbRELAS, 5/40 (12.5\%) UCTs,
11/40 (27.5\%) UELAS, 12/40 (30\%) retrospective case series and $7 / 40(17.5 \%)$ case reports (Table 1). Overall, the 40 selected studies described 10 different AEDs. AEDs were usually given orally in all but three studies, where medication was given intraperitoneally or via transdermal application.

Signalment and baseline characteristics of study subjects Baseline characteristics, i.e. breed, age and sex, of feline population were reported to some degree in all the studies. Signalment was reported in all the clinical studies, with various breeds, both sexes and a wide range of ages at study entry (range $0.25-19$ years) being reported. The most common reported breed was domestic shorthaired cats followed by Birmans, domestic longhaired cats, Siamese, Burmese, Bengals, Himalayan and Maine Coon. Males were more commonly affected compared to females, though these results were not statistically examined in order to specifically report the prevalence of IE on the grounds of sex.

\section{Disease characterization and subject enrolment quality} In approximately half of the studies (19/40, 48\%), the inclusion criteria for diagnosing IE (clinical studies) or healthy cats (ELAS) were not well described (Table 1). Three case reports, each one described an otherwise healthy cat that was suspected with structural epilepsy due to an anesthesia-related hypoxic event [22] or posttramatic/vascular event [23, 24]. However, these studies were included in our review for evaluating the AED safety profile, as their aim was to describe an AEDrelated adverse effect that was unrelated to the cause of the epilepsy.

\section{Study group sizes}

Many studies (36/40, 90\%) assessed small or very small study size groups (Table 1).

\section{Methodological quality of included studies}

Many studies (96\%) showed high or unclear risk of bias (Table 1).

\section{AEDs efficacy and safety profile}

As in our previous systematic reviews [8, 15], details of studies' data, characteristics and outcomes are summarized in the manuscript with further details captured in Additional file 2: Table S1, Tables 2, 3 and 4 and Figs. 1, 2, 3 and 4 .

\section{Phenobarbital}

Eight studies [4, 25-31] assessed the efficacy of phenobarbital as a monotherapy agent or in combination with other AEDs (two studies), providing a total size of 137 cats. Based on the $95 \% \mathrm{CI}$, in all the studies but three 


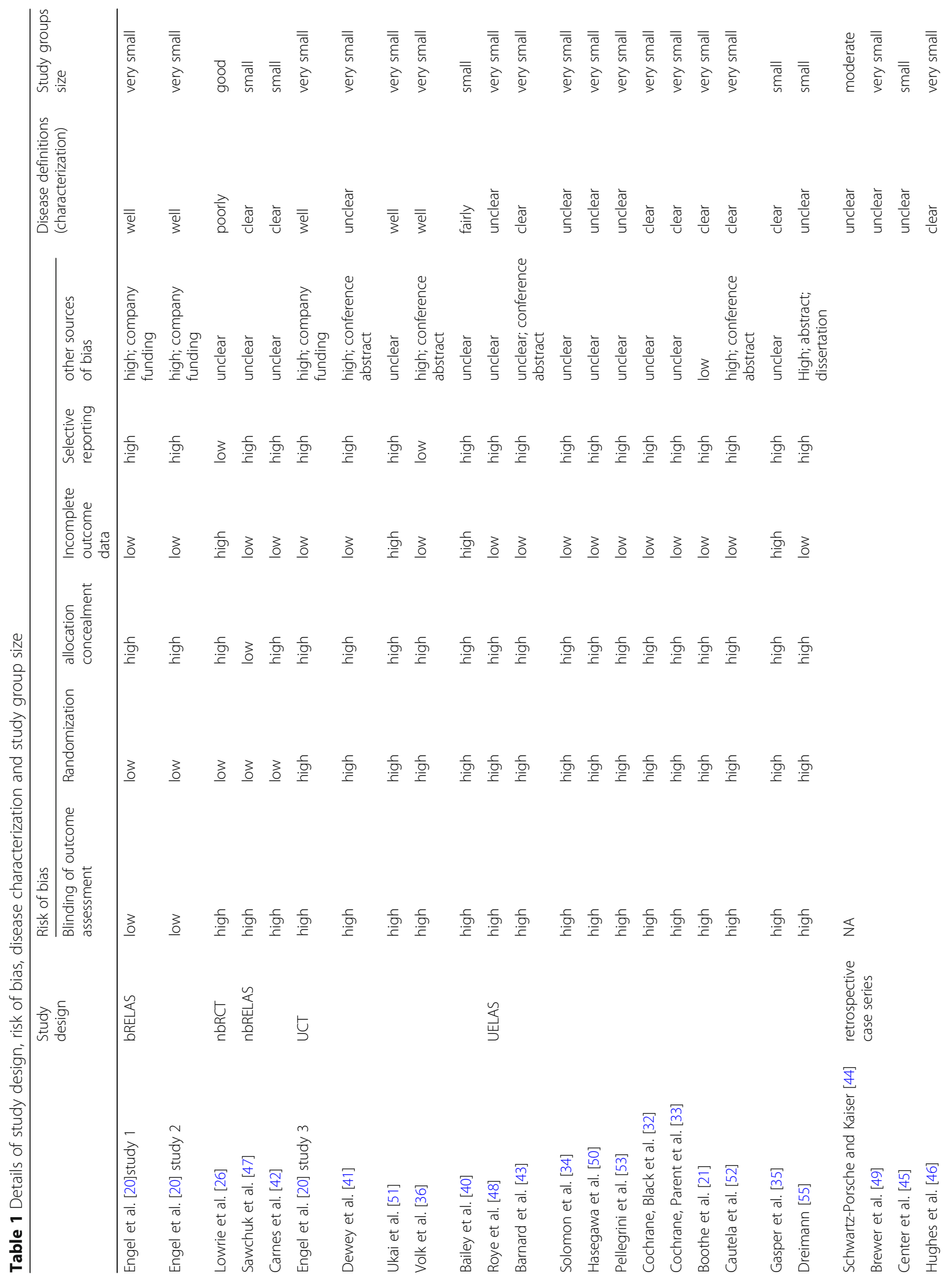




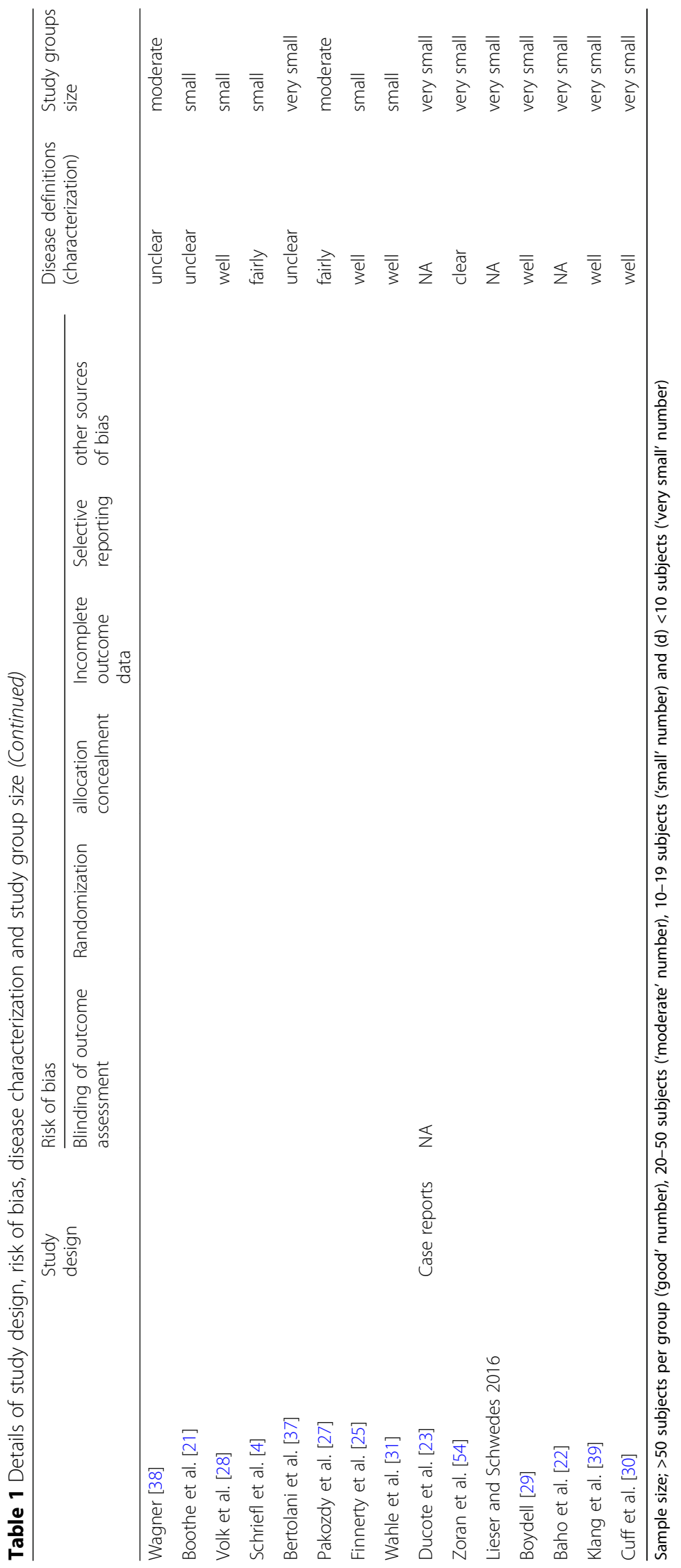


Charalambous et al. BMC Veterinary Research (2018) 14:64

Page 6 of 19

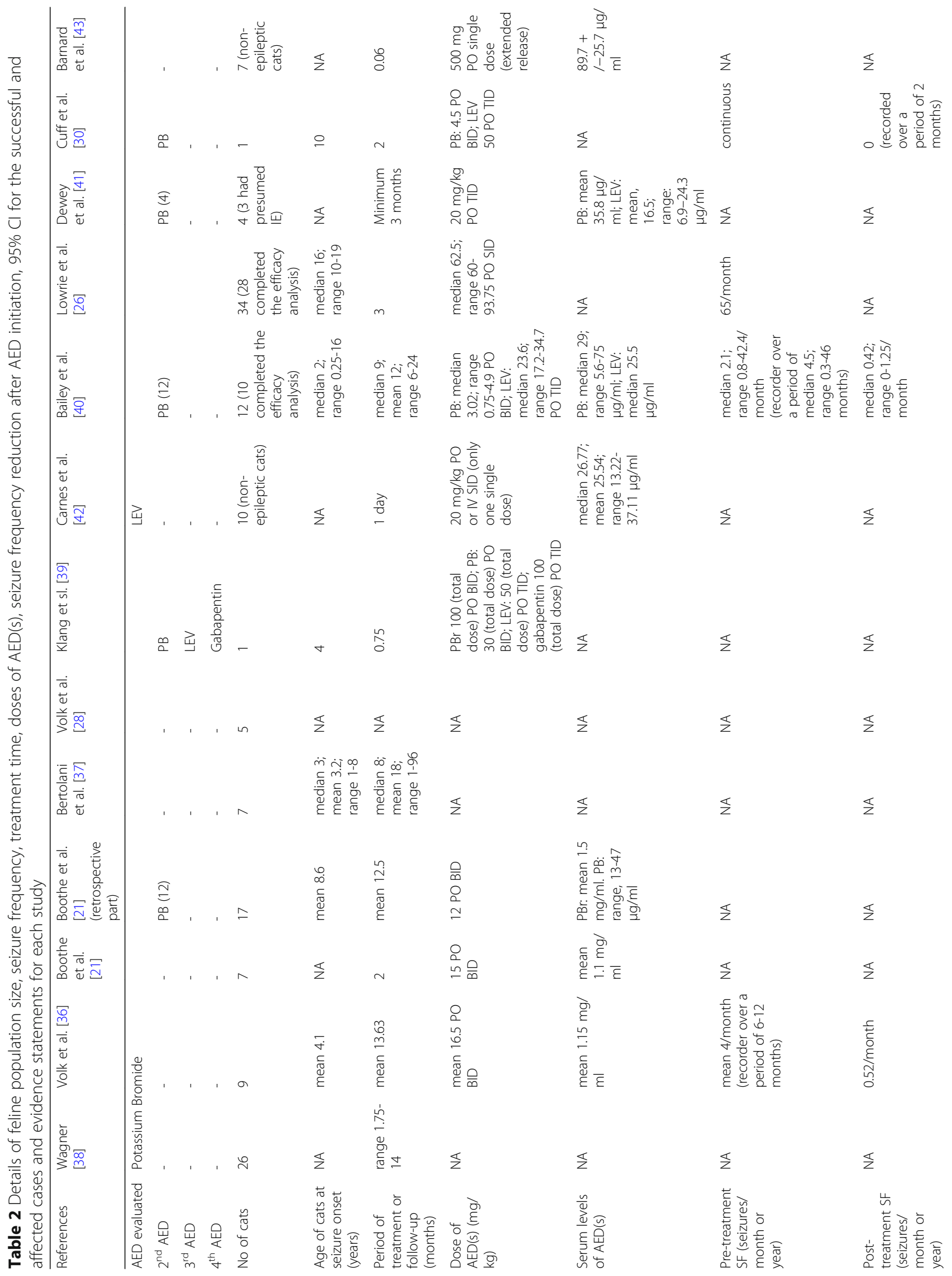


Charalambous et al. BMC Veterinary Research (2018) 14:64

Page 7 of 19

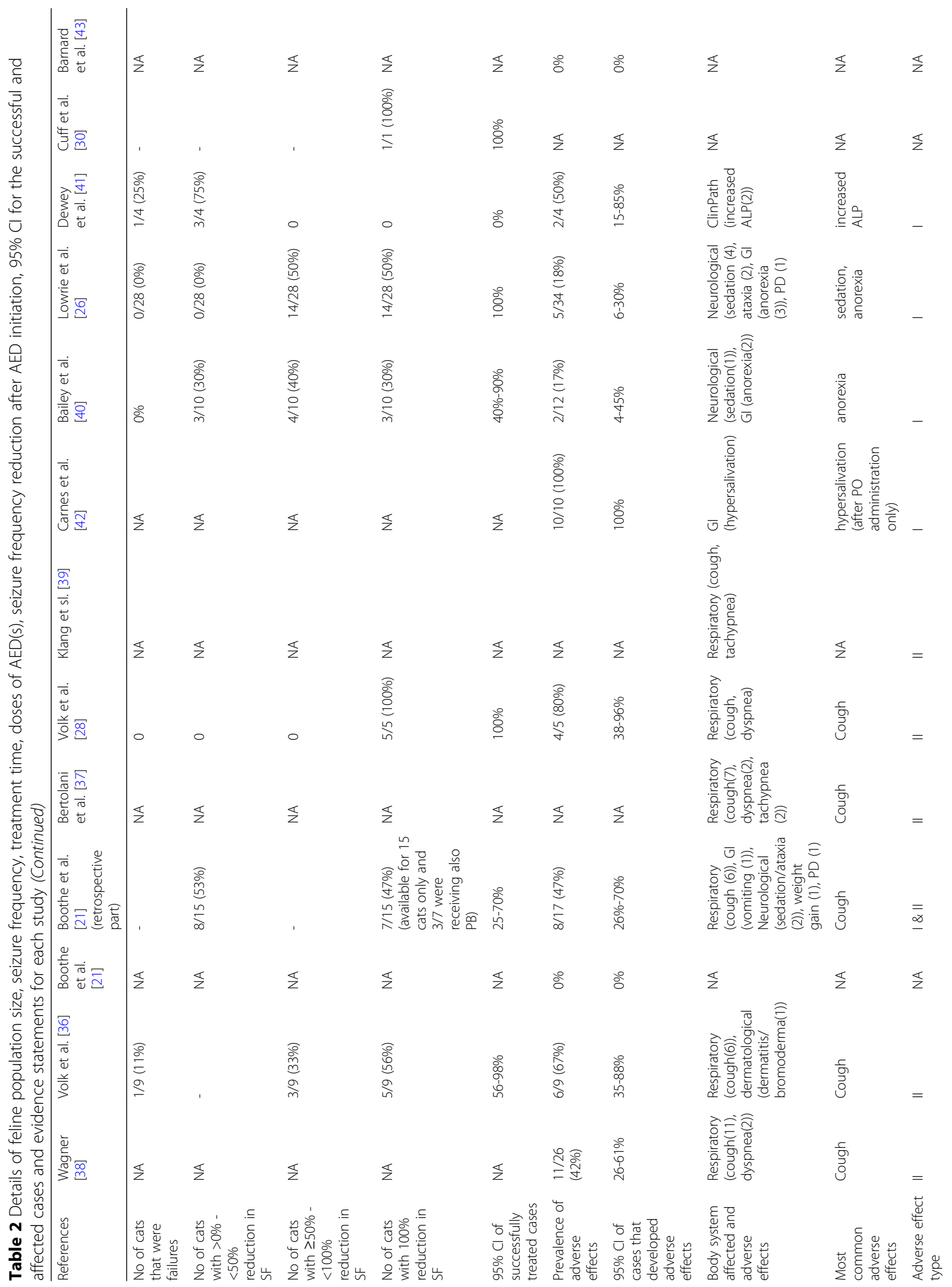




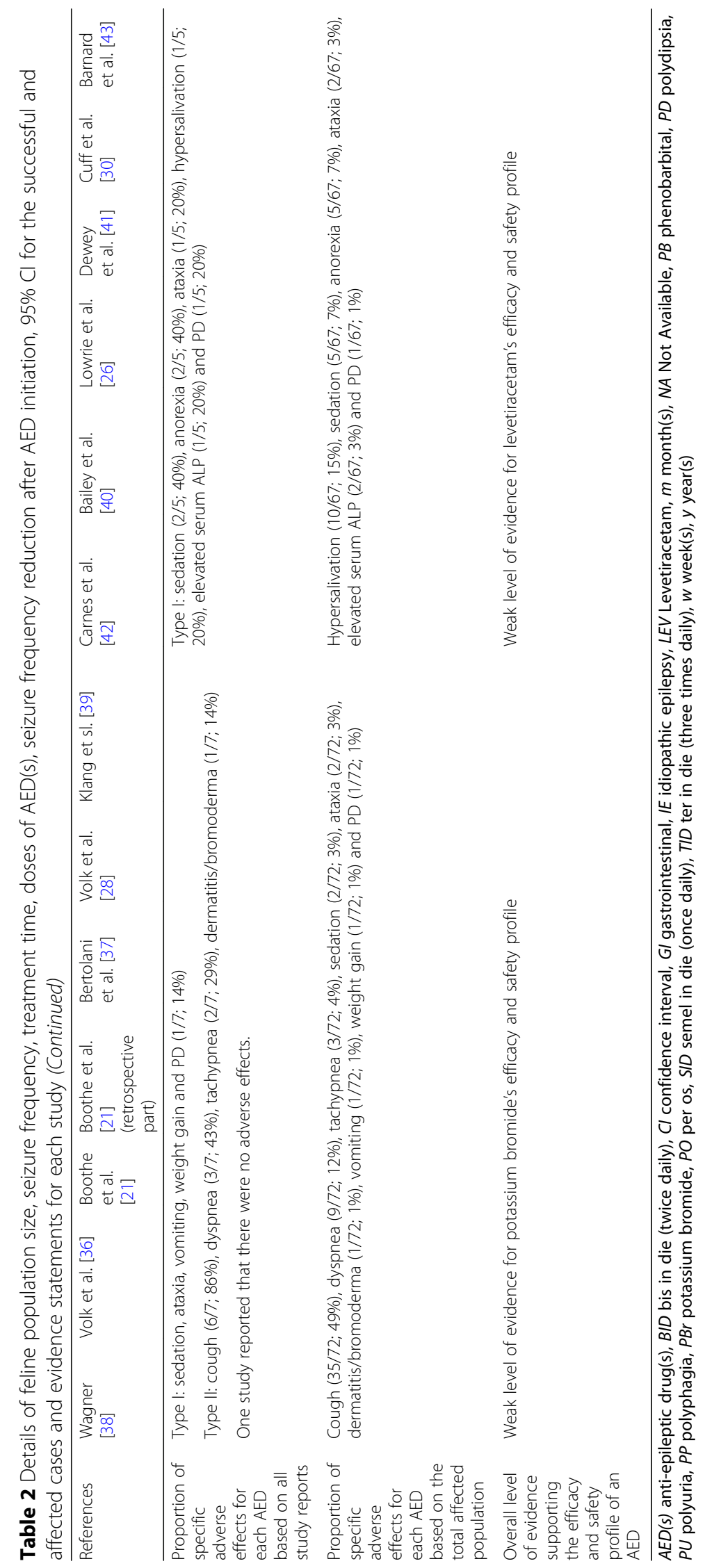


Charalambous et al. BMC Veterinary Research (2018) 14:64

Page 9 of 19

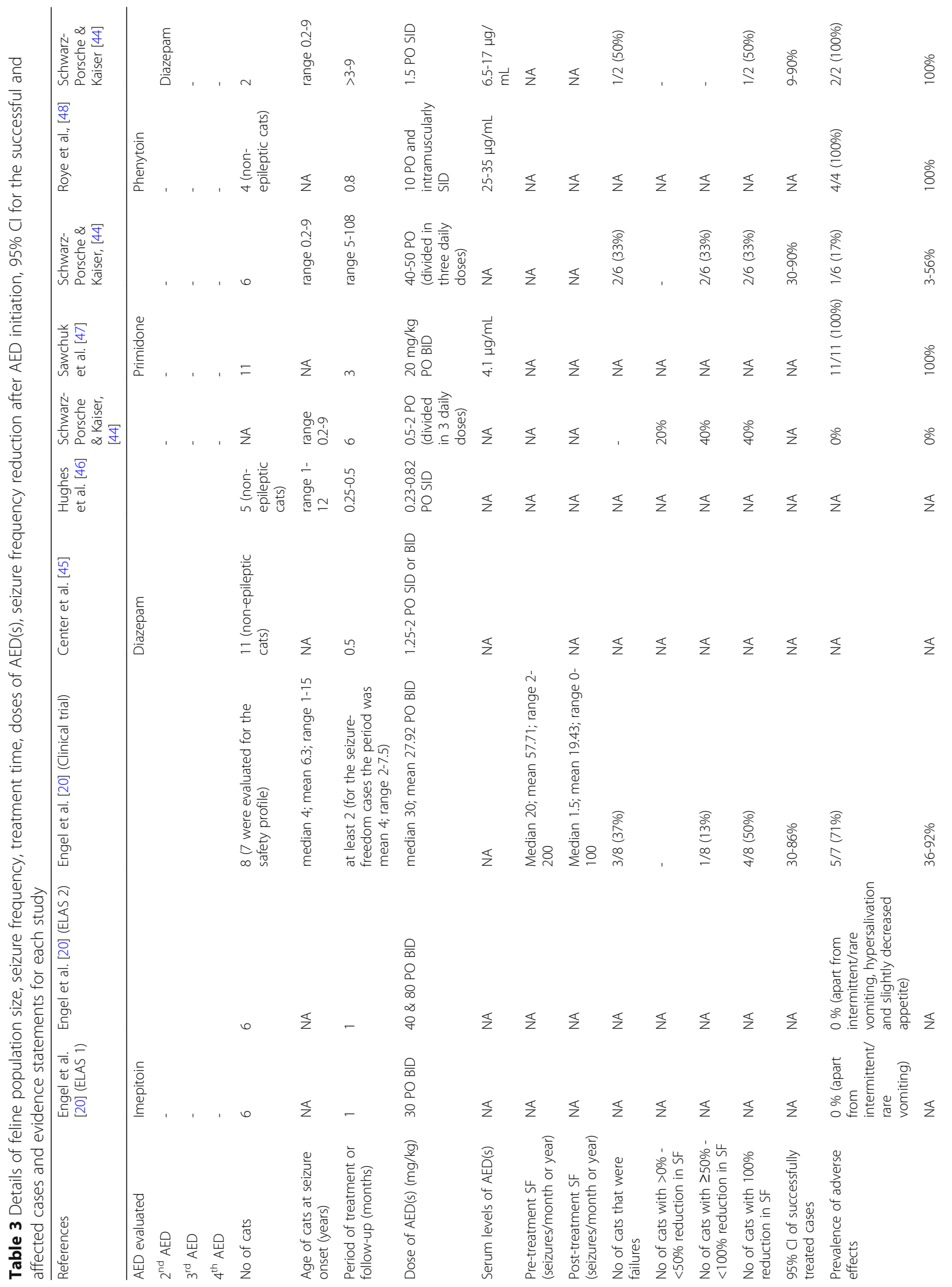




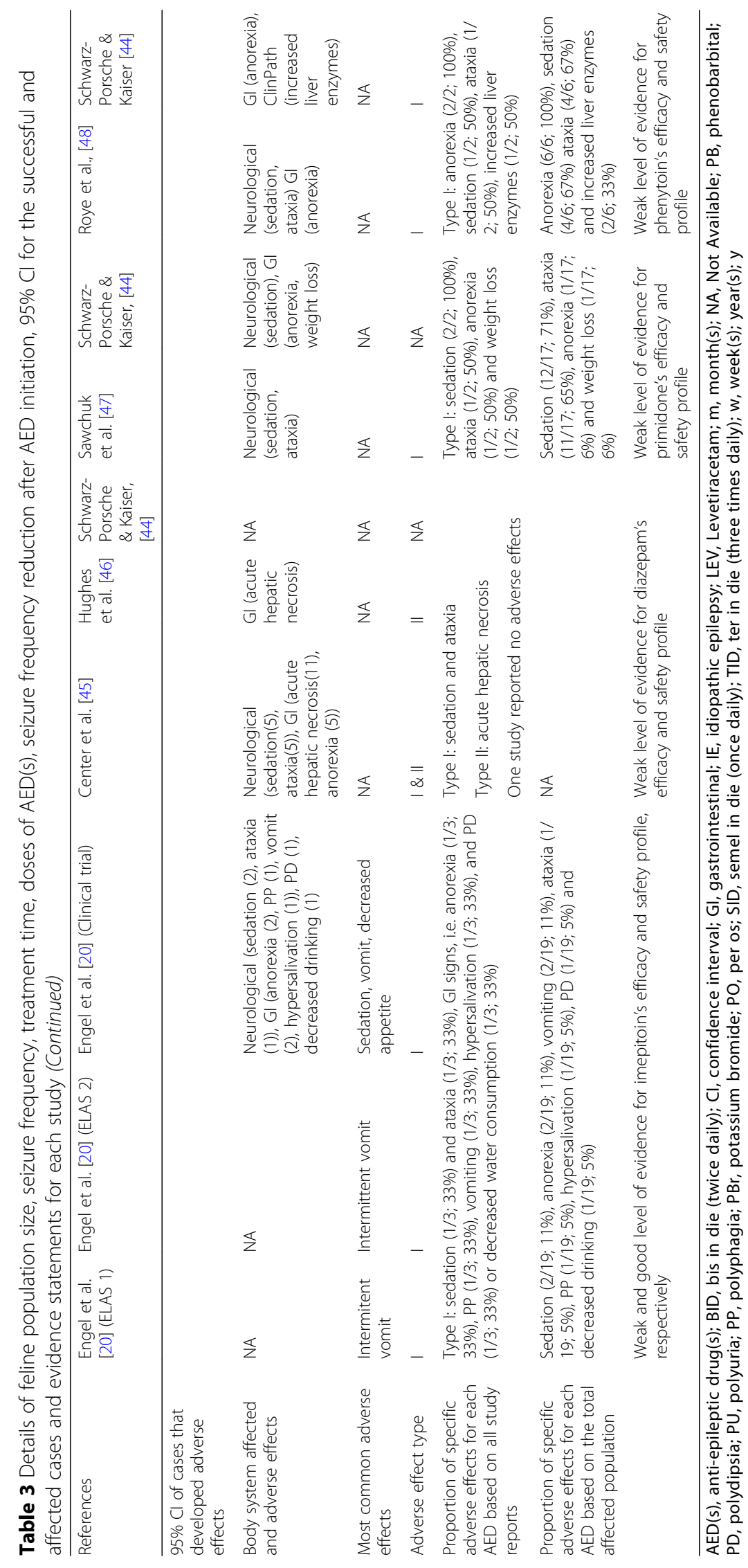




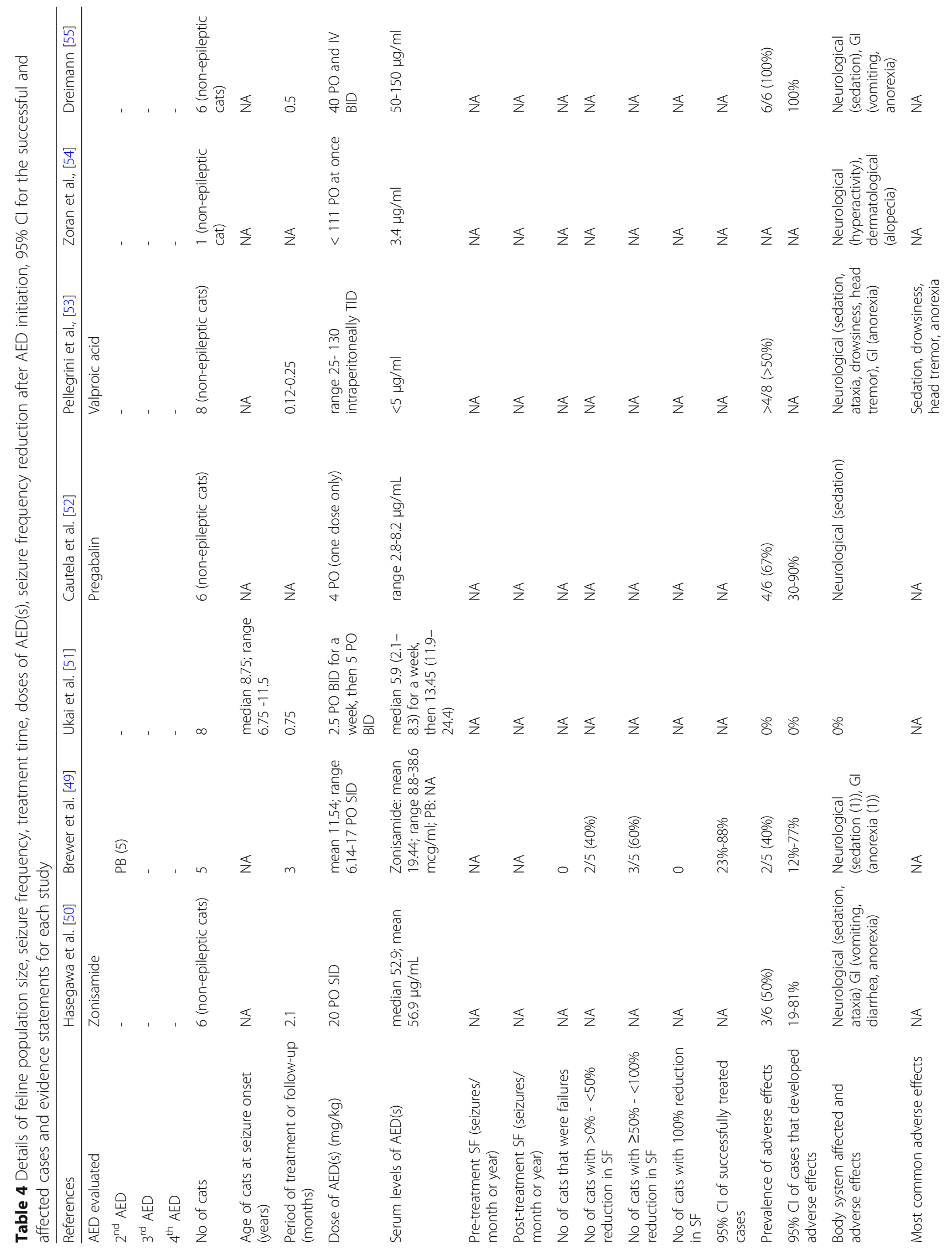




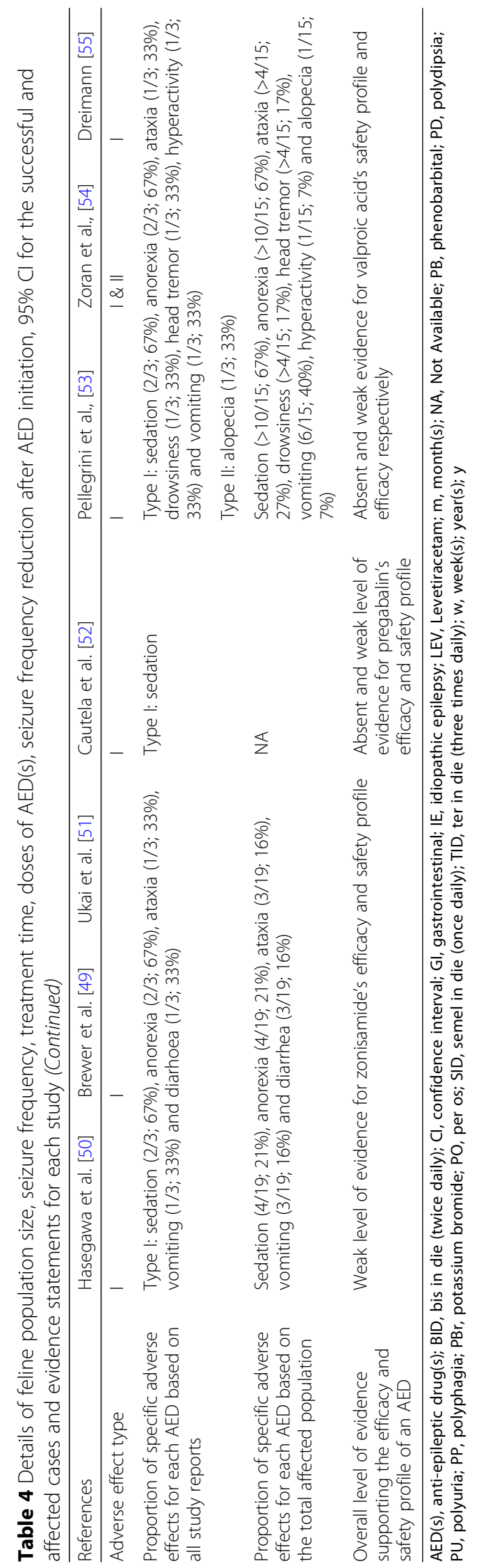




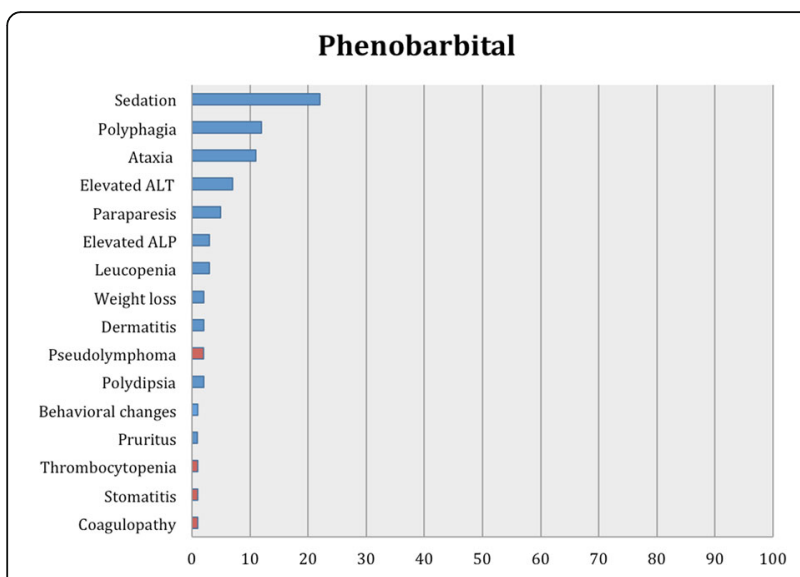

Fig. 1 Proportion of specific adverse effects for phenobarbital. Each adverse effect represents the percentage of cats that were affected by this with regards to the overall combined population for phenobarbital. The blue and red bars indicate type I and II adverse effects, respectively

$[4,26,31](63 \%)$, the majority of the study population was treated successfully. In one of these studies though [26], phenobarbital was only used to treat a specific type of epileptic seizures in cats, i.e. feline auditory reactive seizures (FARS), which might have biased the results for the drug's overall efficacy to control seizures.

Twelve studies [22-28, 31-35] evaluated the safety profile of phenobarbital, providing a total size of 147 cats. Three studies documented type I adverse effects, including dermatological and neurological signs, clinic-pathological abnormalities, polyphagia [36], polydipsia (PD) and weight loss (Additional file 2: Table S1). Four studies reported type II adverse effects including clinic-pathological abnormalities and lympho-reticular and gastrointestinal signs (GI) signs (Additional file 2: Table S1). Three studies reported that there were no adverse effects. Based on the $95 \% \mathrm{CI}$, in all studies but two $[27,34](83 \%)$, the majority of reported

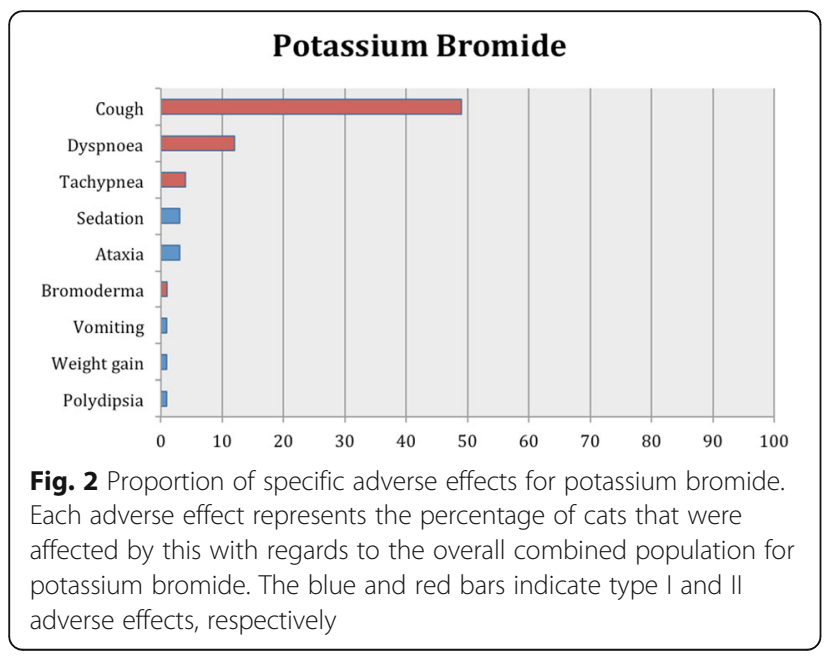

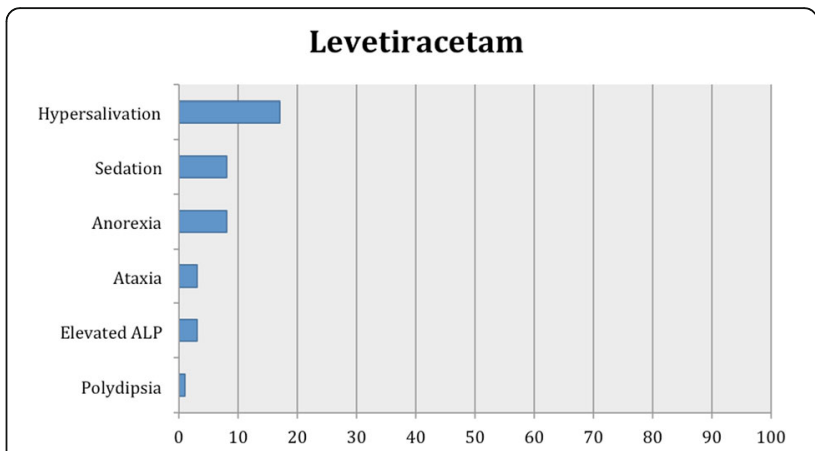

Fig. 3 Proportion of adverse effects for levetiracetam. Each adverse effect represents the percentage of cats that were affected by this with regards to the overall combined population for levetiracetam. The blue bars indicate type I adverse effects

population did not show adverse effects. The most common adverse effect was sedation and the least common were behavioral changes, pruritus, thrombocytopenia, ulcerative stomatitis and coagulopathy (Additional file 2: Table S1, Fig. 1).

The treatment or follow-up period was reported adequately in 14/15 (93\%) studies (Additional file 2: Table S1). From these, in 9/14 (60\%) the follow-up time was short $(<6$ months). Dose and serum levels were provided adequately in $13 / 15(87 \%)$ and $11 / 15(73 \%)$ studies respectively (Additional file 2: Table S1). The phenobarbital maintenance doses and serum levels were higher than the normal reference range [6] in $8 / 13(62 \%)$ and $4 / 11$ (36\%) studies respectively. In all, type I and II adverse effects did not follow a dose or serum levels dependent pattern. In summary, the level of evidence for phenobarbital's efficacy and safety profile was weak.

\section{Potassium Bromide}

Three studies [21, 28, 36] assessed potassium bromide's efficacy as a monotherapy agent or in combination to phenobarbital (one study), providing a total group size

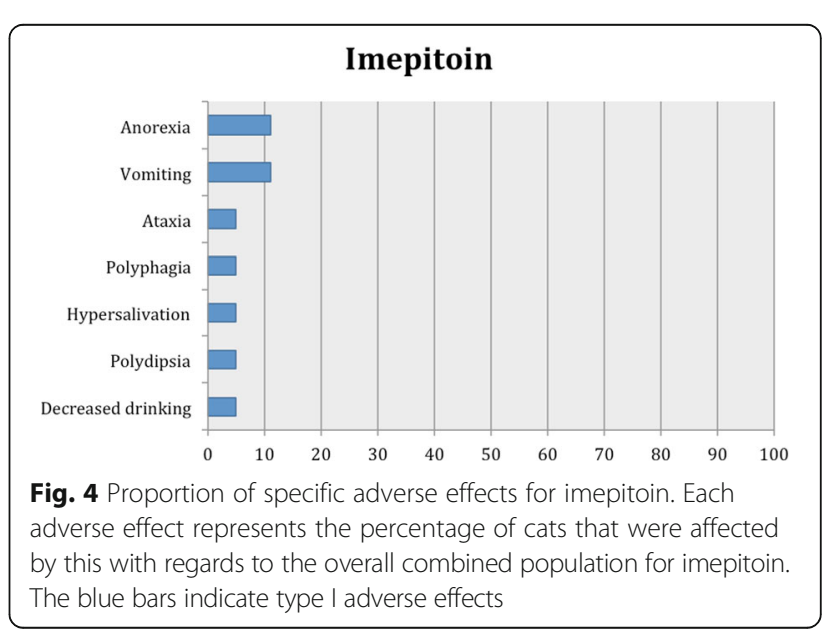


of 29 cats. Based on the $95 \% \mathrm{CI}$, in all the studies but one [21] (66\%), the majority of the study population was treated successfully.

Six studies [21, 28, 36-39] evaluated the safety profile of potassium bromide, providing a total size of 72 cats. One of these studies included both a UCT and retrospective case series part [21]. One study documented type I adverse effects, such as neurological, GI signs and PD. Five studies reported type II adverse effects, such as respiratory and dermatological signs (Table 2). One study reported that there were no adverse effects. Applying the $95 \% \mathrm{CI}$, in all the studies (100\%), the majority of the cases reported did not have adverse effects. The most common adverse effect was cough and least common were dermatitis/bromoderma, vomiting, weight gain and PD (Table 2, Fig. 2).

Treatment or follow-up time was reported adequately in $6 / 7(86 \%)$ (Table 2). From these, in $2 / 6(33 \%)$ the period was short ( $<6$ months). The dose and serum levels were documented adequately in $4 / 7$ (57\%) and 3/7 (43\%) studies (Table 2). There was no adequate information to relate specific range values of dose and serum levels with the development of type I or II adverse effects. In summary, the level of evidence for potassium bromide's efficacy and safety profile was weak.

\section{Levetiracetam}

Four studies $[26,30,40,41]$ assessed the efficacy of levetiracetam as a monotherapy agent or in combination to phenobarbital (three studies), providing a total group size of 43 cats. Based on the $95 \%$ CI, in two of the studies $[26,30](50 \%)$, the majority of the study population was treated successfully.

Five studies [26, 40-43] reported the safety profile of levetiracetam, providing a total group size of 67 cats. Only type I adverse effects were reported in all evaluated studies, including neurological and GI signs and clinicopathological abnormalities (Table 2). Based on the 95\% CI, in all of the studies but one [42] (75\%), the majority had no adverse effects reported. One study reported no adverse effects [43]. The most common adverse effect was hypersalivation and the least common was PD (Table 2, Fig. 3).

The treatment or follow-up time was reported adequately in all the studies (Table 2), but was short ( $<6$ months) in $5 / 6(83 \%)$. The dose and serum levels were reported adequately in $6 / 6(100 \%)$ and $4 / 6(66 \%)$ studies respectively (Table 2). The levetiracetam maintenance doses reported were higher than normal in 2/6 (33\%) studies. In all, type I adverse effects did not follow a dose or serum levels dependent pattern. The level of evidence for levetiracetam's efficacy and safety profile was weak.

\section{Imepitoin}

One study [20] assessed the efficacy of imepitoin as monotherapy agent in 8 cats. With such a small population size studied the $95 \%$ CI could not demonstrate that the majority of cases were managed successfully.

Three studies, which were included as part of one report [20], reported the safety profile of imepitoin, providing a total study size of 19 cats. In one study, only type I adverse effects were reported including neurological, GI signs and $\mathrm{PD}$ or decreased water consumption. In the two other studies though, no such effects were reported but intermittent vomiting, hypersalivation and slightly decreased appetite (Table 3). Applying the 95\% CI to all study data $(100 \%)$, the majority of cases did not experience adverse effects. There was an equal distribution among all the adverse effects (Table 3, Fig. 4).

The treatment or follow-up time was reported adequately in all studies (Table 3$)$. In all studies $(100 \%)$ the period was short ( $<6$ months). The dose and serum levels was provided adequately in $3 / 3(100 \%)$ and $0 / 3(0 \%)$ studies, respectively (Table 3 ). Higher imepitoin maintenance doses, i.e. 40 or $80 \mathrm{mg} / \mathrm{kg}$ PO BID, were associated with higher incidence of adverse effects. The level of evidence for imepitoin's efficacy was weak but the level of evidence for safety profile was strong.

\section{Diazepam}

One study [44] assessed the efficacy of diazepam as monotherapy. The study reported that the majority of the cases was managed successfully, but the 95\% CI could not be calculated.

Three studies [44-46] reported the safety profile of diazepam, providing a total size of 16 cats. One study documented type I adverse effects, i.e. neurological signs (Table 3). Two studies documented type II adverse effects, i.e. GI signs (Table 3). One study reported that there were no adverse effects. Since all the studies, but one [44], selectively included cats that manifested adverse effects, the 95\% CI and adverse effects prevalence could not be calculated (Table 3).

The treatment or follow-up time was reported adequately in $3 / 3(100 \%)$, but was considered short in all (Table 3). The dose and serum levels were reported adequately in $3 / 3(100 \%)$ and $0 / 3(0 \%)$ studies respectively (Table 3). In all, type I and II adverse effects did not follow a dose or serum levels dependent pattern. The level of evidence for diazepam's efficacy and safety profile was weak.

\section{Primidone}

One study [44] assessed the efficacy of primidone as a monotherapy agent in 6 cats. Applying the 95\% CI to such a small study population showed that the majority was not managed successfully. 
Two studies [44, 47] reported the safety profile of primidone, providing a total group size of 17 cats. The studies reported type I adverse effects, such as GI and neurological signs and weight loss. No type II adverse effects were reported (Table 3). Applying the 95\% CI to one study [44] (50\%), the majority of cases did not have adverse effects reported. The most common adverse effect was sedation and the least common were anorexia and weight loss (Table 3).

The treatment or follow-up period was reported adequately in $2 / 2(100 \%)$ (Table 3 ). From these, in $1 / 2$ (50\%) the period was short ( $<6$ months). The dose and serum levels was reported adequately in $2 / 2(100 \%)$ and $1 / 2(50 \%)$ studies respectively (Table 3 ). There was inadequate information to relate specific range values of dose and serum levels with the development of type I or II adverse effects. The level of evidence for primidone's efficacy and safety profile was weak.

\section{Phenytoin}

One study [44] evaluated the efficacy of phenytoin as an adjunct to diazepam in 2 cats.

Two studies [44, 48] reported the safety profile of phenytoin, providing a total size of 6 cats. The studies documented type I adverse effects, such as GI and neurological signs and clinic-pathological abnormalities. No type II adverse effects were documented (Table 3). Based on the $95 \% \mathrm{CI}$, in all the studies (100\%), the majority of the study population experienced adverse effects. The most common adverse effect was anorexia and the least common was increased serum liver enzymes (Table 3 ).

The treatment or follow-up period was reported adequately in $2 / 2(100 \%)$ (Table 3$)$. From these, in $1 / 2(50 \%)$ the period was short ( $<6$ months). The dose and serum levels were reported adequately in $2 / 2(100 \%)$ studies (Table 3). There was inadequate information to relate specific range values of dose and serum levels with the development of type I or II adverse effects. The level of evidence for phenytoin's efficacy and safety profile was weak.

\section{Zonisamide}

One study [49] assessed the efficacy of zonisamide as an adjunct to phenobarbital in 5 cats. Applying the 95\% CI to such a small study population showed that the majority was not managed successfully.

Three studies [49-51] reported the safety profile of zonisamide in 19 cats. The studies documented type I adverse effects, such as GI and neurological signs (Table 4). Based on the 95\% CI, in all studies (100\%), the majority of the study population did not have adverse effects. One study reported no adverse effects [51]
The treatment or follow-up time was reported adequately but was considered short in all studies (Table 4). The dose and serum levels were also reported adequately in all studies (Table 4). There was inadequate information to relate specific range values of dose and serum levels with the development of type I or II adverse effects. In summary, the level of evidence for zonisamide's efficacy and safety profile was weak.

\section{Pregabalin}

There were no original studies evaluating the efficacy of pregabalin in cats except for experts' opinion. One study [52] reported the safety profile of pregabalin in 6 cats. The study documented type I adverse effects, i.e. neurological signs. No type II adverse effects were documented (Table 4). Applying the $95 \%$ CI to the studied population, the majority did not experience adverse effects.

The treatment or follow-up time was inadequately reported (Table 4). The dose and serum levels was reported adequately (Table 4). There was inadequate information to relate specific range values of dose and serum levels with the development of type I or II adverse effects. There was no evidence for pregabalin's efficacy and an overall weak level of evidence for pregabalin's safety profile.

\section{Valproic acid}

There were no original studies evaluating the efficacy of valproic acid in cats except for experts' opinion. Three studies [53-55] reported the safety profile of valproic acid, giving a combined sample size of 15 cats. Three studies documented type I adverse effects, such as GI and neurological signs. One study documented type II adverse effects, i.e. dermatological signs (Table 4). There was inadequate information to calculate the $95 \%$ CI but one study [55] showed that the whole population had adverse effects. The most common adverse effects were sedation ataxia, drowsiness, head tremor and anorexia and the least common were hyperactivity and alopecia.

The treatment or follow-up time was reported adequately in $2 / 3(67 \%)$ studies and was considered short. The dose and serum levels were reported adequately in $3 / 3(100 \%)$ (Table 4). There was inadequate information to relate specific range values of dose and serum levels with the development of type I or II adverse effects. There was no evidence for valproic acid's efficacy and weak level of evidence for safety profile.

\section{Discussion}

To the authors' knowledge, this is the first systematic review of AEDs' efficacy and safety in cats. The authors were based on the PRISMA (Preferred Reporting Items for Systematic Reviews and Meta-Analyses) statement to report this systematic review [56]. The systematic review 
found that the level of evidence in feline epilepsy treatment is weak to absent, in particular for AEDs' efficacy. The results showed that phenobarbital was considered the most effective AED followed by levetiracetam and potassium bromide, then imepitoin and diazepam and lastly zonisamide, primidone and phenytoin (Fig. 5). All supported by weak level of evidence. There was insufficient evidence for the efficacy of pregabalin and valproic acid. As far as the safety profile of all the AEDs is concerned, imepitoin was considered the safest AED, followed by levetiracetam and phenobarbital, then zonisamide and pregabalin followed by primidone, phenytoin and valproic acid and lastly potassium bromide and diazepam (Fig. 6). All supported by weak level of evidence apart from imepitoin, which was supported by good level of evidence. Although this systematic review focused on evaluating the use of AEDs in feline patients, it would be an omission not to mention that other substances have been proposed for managing seizures in cats $[57,58]$, with taurine as the most representative example. In a feline case report, administration of $300 \mathrm{mg}$ taurine subcutaneously twice a day for two days followed by $100 \mathrm{mg}$ taurine orally once a day for one month resulted in reduction in seizure frequency, supported by clinical and electroencephalographic observations. Abrupt cessation led to a rise of frequency [57]. Further clinical studies are crucial to support taurine's potential efficacy in feline epilepsy.

The AEDs' adverse effects were categorised as type I (predictable or dose-dependent) or type II (idiosyncratic/ unpredictable or dose-independent) which are usually considered as non-immunological reactions [59]. It was challenging to detect a correlation between the AED dose or serum concentration and the type of adverse effects in feline patients. This could be attributed either to the possibility that, in feline patients, adverse effects might not follow a dose or serum related pattern or to the fact that there was inadequate information to allow us assessing any potential correlation between the incidence of adverse effects and the AEDs' dose or serum

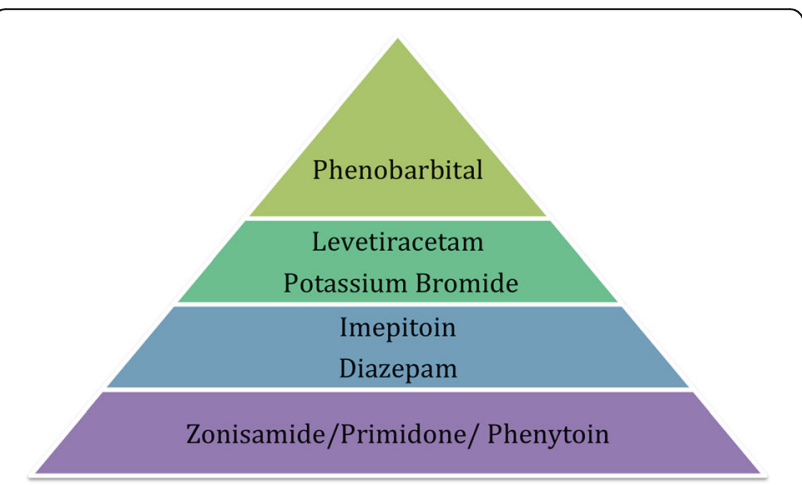

Fig. 5 Pyramid of AEDs' efficacy hierarchy based on the quality of evidence and outcomes assessment

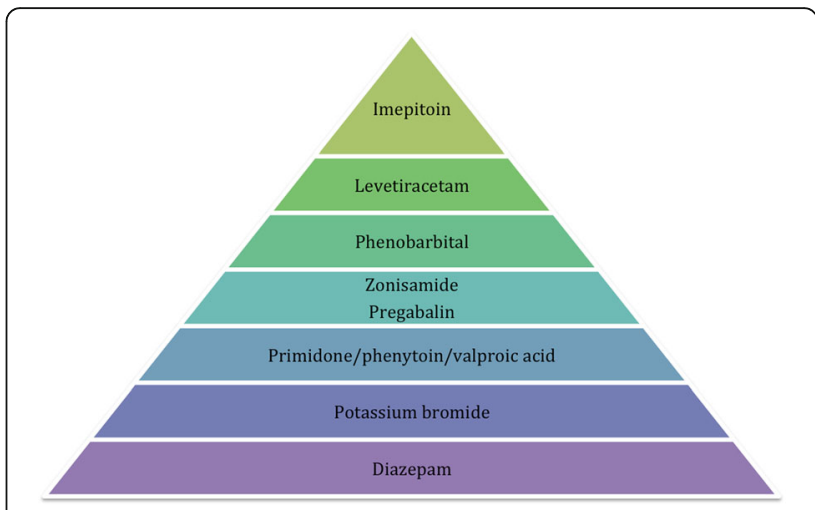

Fig. 6 Pyramid of AEDs' safety hierarchy based on the quality of evidence and outcomes assessment

concentrations. Severe type II adverse effects were reported mainly in cats being treated with potassium bromide or diazepam. Type II adverse effects are non-dose dependent, unpredictable, usually rare and caused by a cytotoxin, a drug or its metabolite [59]. Idiosyncratic reactions are most likely secondary to an individual difference in rate of formation and detoxification of reactive metabolites [59]. However, in the case of potassium bromide and, in particular, diazepam in feline patients, the incidence of these type II effects was high and in case of diazepam, they were considered life-threatening. This can give rise to evidence that the type II adverse effects of these two drugs in cats are species-related rather than individual-related.

In canine epilepsy, the majority of studies lacked a high level of evidence $[8,15]$. However, in feline epilepsy, almost none of the studies provided high quality of evidence. The studies manifested high overall risk of bias. In addition, only $47 \%$ and $2 \%$ of all studies had well clinically defined groups and assessed a sufficient number of cats, respectively. The $95 \% \mathrm{CI}$, that was used as an indicator of the 'real' population of successful (AED efficacy) or affected (AED safety) cases, revealed a wide range of values mainly due to the inadequate feline population in the studies. Therefore, conclusions drawn based on the $95 \%$ CI results should be interpreted with caution. In addition, there were no bRCTs evaluating any AEDs in cats but two bRELAS assessing imepitoin's safety profile. The follow up time was rather limited and short $(<6$ months $)$ to assess adequately long-term efficacy and tolerability. In all, due to the lack of studies with overall low risk of bias, insufficient disease characterisations and small group sizes, clear suggestions concerning AEDs' efficacy and safety are difficult.

In contrast to canine epilepsy, many studies evaluated the efficacy and safety of an AED as monotherapy (with the exception of levetiracetam), making it easier to determine whether an AED's adverse effects and efficacy were attributed mainly to its administration. However, 
certain evaluated factors might have influenced our assessment of AEDs' efficacy and safety profile similar to the ones described in our previous systematic reviews for canine patients $[8,15]$. These included the dosages used of a particular AED (i.e. different among studies), the frequency of AEDs' administration (i.e. factor that could alter the chances of occurrence of adverse effects particularly in AEDs with short half-life due to fluctuations of serum drug levels; although this characteristic might not affect the results [60]), the duration of the study (i.e. insufficient duration, as it was found in many studies, might have reduced the chance for the most frequent adverse effects to occur or for adequately evaluate an AED's efficacy). Lastly, a few AEDs have been used more often in feline epilepsy and been in the market for longer period compared to others. Therefore, more evidence is available which could influence the conclusions with regards to their efficacy and safety. A characteristic example is phenobarbital for which, compared to other AEDs, there are more reports of not only its efficacy but also its adverse effects.

As it was found in the previous canine systematic reviews and meta-analysis [8, 15], some characteristics may have also adversely affected the evaluation of the included studies. Similarly, multiple factors could have influenced our results such as signalment differed between studies, heterogeneity in treatment methods among studies, variation in study publication dates, publication bias, several introduced biases detected, lack of high quality evidence studies (i.e. bRCTs and bRELAS), lack of well characterized diagnostic procedures and enrolment of relatively small study population.

\section{Implications for research}

Systematic reviews are a good step towards clinical evidence medicine, however the evaluation and comparison among AEDs through a meta-analysis could provide far further information and aid clinician's decision to choose the most appropriate AED for every patient. A metaanalysis was not feasible here due to the variations in baseline characteristics of the cats involved, the significant differences between study designs, the several sources of identified bias and mainly due to the lack of comparison group studies. Although the $95 \% \mathrm{CI}$ and the prevalence of successfully treated cases and adverse effects in each study provide a general indicator of each AED's efficacy and safety profile, respectively, and can lead to indirect comparisons between AEDs, comparison group studies are essential as they allow thorough statistics to be performed for direct and thus more reliable comparisons. Therefore, further comparison groups and blinded randomized studies are essential for feline epilepsy treatment that would allow a meta-analysis towards this goal.
Lastly, a further problem that was found during the studies evaluation was the lack of reported information. This led to difficulties in performing statistical analysis for AEDs' comparisons. Therefore, it is essential that future trials should provide precise information and scientists have open access to trials' data. It would be crucial that journals enforce authors to report their results based on guidelines such as STROBE (Strengthening the Reporting of Observational studies in Epidemiology), ARRIVE (Animal Research: Reporting of In Vivo Experiments) and CONSORT (Consolidated Standards of Reporting Trials).

\section{Implications for clinical practice}

Current evidence did not allow comparisons among AEDs, and therefore it would be rather inaccurate to make definite statements on which one should be considered as a first or second choice in terms of both efficacy and safety profile. However, if clinicians focus on AED's efficacy, phenobarbital can be used as first-choice monotherapy and if they focus on AED's safety, imepitoin or levetiracetam can be used. It is important to report that, similarly to canine epilepsy [8], phenobarbital should not be overstated as an AED with high incidence of detrimental adverse effects and therefore, in healthy cats with no pre-existing liver disease, phenobarbital might be the most appropriate choice. Levetiracetam or imepitoin can also be considered as a safe alternative for monotherapy, especially in cats that develop unexpected adverse effects to phenobarbital. For certain epilepsy phenotypes, such as myoclonic epilepsy in elderly cats, levetiracetam monotherapy can be considered. Levetiracetam, imepitoin or phenorbarbital and, to a quite lesser degree, zonisamide might be considered as add-on medication when first line treatment chosen is not sufficient to control seizures. Potassium bromide can be used as adjunctive AEDs only as a last resort and after a signed owner's consent form in cases manifesting resistance to $>2$ AEDs and should be closely monitored for type II adverse effects. Diazepam might not be an appropriate choice due to the inadequate evidence supporting its efficacy and the possibility of severe and potentially life-threatening type II adverse effects. It is also important to note that the recommendations made are on the basis of the evidence provided in the available literature and local drug legislation need to be considered prior of prescribing medications.

In general, as it was found in our previous systematic review [8], most of the AED adverse effects documented were inconsistent, fairly tolerable and not lifethreatening and ceased once doses and serum levels were decreased or following drug withdrawal. Exceptions included specific type II adverse effects and specific antiepileptic drugs (in particular diazepam and to a lesser degree potassium bromide). It is essential that clinicians 
assess both the benefits (i.e. value for money, dosing regimen, efficacy) and risks (i.e. safety and tolerability, and impact of adverse effects on the cat's and owner's quality of life) before choosing a specific AED.

\section{Conclusion}

This systematic review provides an evidence-based assessment of the data on the AEDs' efficacy and adverse effects for feline epilepsy. Factors that need to be considered when evaluating these results are: i) drugs that have been used and been on the market for longer periods provided more evidence, ii) the vast majority of the studies offered overall high risk of bias and included small number of cats with unclear or fair disease characterization criteria and short-term follow-up. Individual AED assessments of efficacy and safety profile showed that phenobarbital can be used as the first-choice AED followed by imepitoin and levetiracetam. Further studies in feline epilepsy treatment are by far essential in order to establish definite guidelines for AEDs' efficacy and safety.

\section{Additional file}

Additional file 1: PRISMA flow diagram. (DOC $56 \mathrm{~kb}$ )

Additional file 2: Table S1. Details of feline population size, seizure frequency, treatment time, doses of $A E D(s)$, seizure frequency reduction after AED initiation, 95\% Cl for the successful and affected cases and evidence statements for each study. (DOCX $32 \mathrm{~kb}$ )

\section{Abbreviations}

AED(s): anti-epileptic drug(s); BID: bis in die (twice daily); bELAS: blinded experimental laboratory animal studies; bRCTs: blinded randomised clinical trials; Cl: confidence interval; CTs: clinical trials; Gl: gastrointestinal; IE: idiopathic epilepsy; LEV: levetiracetam; m: month(s); MRI: magnetic resonance imaging; NA: Not Available; nbELAS: non-blinded experimental laboratory animal studies; nbRCTs: non-blinded randomised clinical trials; NRELAS: non-randomised experimental laboratory studies; NRCTs: non-randomised clinical trials; PB: phenobarbital; PBr: potassium bromide; PD: polydipsia; PO: per os; PP: polyphagia; PU: polyuria; SID: semel in die (once daily); TID: ter in die (three times daily); UCTs: uncontrolled clinical trials; UELAS: uncontrolled experimental laboratory studies; w: week(s)

\section{Acknowledgements}

Not applicable.

\section{Funding}

Not applicable.

\section{Availability of data and materials}

All data generated or analysed during this study are included in this published article.

\section{Authors' contributions}

MC and SB collected the data. MC screened and evaluated the data and drafted the manuscript. AP and HV helped to draft the manuscript and participated in its design. All authors read and approved the final manuscript.

\section{Ethics approval and consent to participate}

Not applicable

\section{Consent for publication}

Not applicable

\section{Competing interests}

$\mathrm{HV}$ is a member of the editorial board of the journal.

\section{Publisher's Note}

Springer Nature remains neutral with regard to jurisdictional claims in published maps and institutional affiliations.

\section{Author details}

${ }^{1}$ Small Animal Department, Faculty of Veterinary Medicine, Ghent University, Merelbeke, Belgium. ${ }^{2}$ Clinical Unit of Internal Medicine Small Animals, University of Veterinary Medicine, Vienna, Austria. ${ }^{3}$ Department of Clinical Science and Services, Royal Veterinary College, Hawkshead Lane, Brookmans Park, UK.

Received: 11 October 2017 Accepted: 21 February 2018

Published online: 02 March 2018

\section{References}

1. Schwartz-Porsche D. Epidemiological, clinical and pharmacokinetic studies in spontaneously epileptic dogs and cats. In: The 4th Annual American College of Veterinary Internal Medicine Forum: 1986. Washington, DC: JVIM; 1986. p. 61-3.

2. Pakozdy A, Leschnik M, Sarchahi AA, Tichy AG, Thalhammer JG. Clinical comparison of primary versus secondary epilepsy in 125 cats. J Feline Med Surg. 2010;12(12):910-6.

3. Cizinauskas S, Fatzer R, Schenkel M, Gandini G, Jaggy A. Can idiopathic epilepsy be confirmed in cats? Journal of Veterinary Internal Medicine. 2003;17:246.

4. Schriefl S, Steinberg TA, Matiasek K, Ossig A, Fenske N, Fischer A. Etiologic classification of seizures, signalment, clinical signs, and outcome in cats with seizure disorders: 91 cases (2000-2004). J Am Vet Med Assoc. 2008;233(10):1591-7.

5. Quesnel AD, Parent JM, McDonell W, Percy D, Lumsden JH. Diagnostic evaluation of cats with seizure disorders: 30 cases (1991-1993). J Am Vet Med Assoc. 1997;210(1):65-71.

6. Bailey KS, Dewey CW. THE SEIZURING CAT Diagnostic work-up and therapy. Journal of Feline Medicine and Surgery. 2009;11(5):385-94.

7. Pakozdy A, Halasz P, Klang A. Epilepsy in cats: theory and practice. J Vet Intern Med. 2014;28(2):255-63.

8. Charalambous M, Shivapour SK, Brodbelt DC, Volk HA. Antiepileptic drugs' tolerability and safety-a systematic review and meta-analysis of adverse effects in dogs. BMC Vet Res. 2016;12:79.

9. Papanikolaou PN, loannidis JP. Availability of large-scale evidence on specific harms from systematic reviews of randomized trials. The American journal of medicine. 2004;117(8):582-9.

10. Ross SD. Drug-related adverse events: a readers' guide to assessing literature reviews and meta-analyses. Archives of internal medicine. 2001;161(8):1041-6.

11. Chou R, Helfand M. Challenges in systematic reviews that assess treatment harms. Annals of internal medicine. 2005;142(12 Pt 2):1090-9.

12. Berlin JA, Colditz GA. The role of meta-analysis in the regulatory process for foods, drugs, and devices. Jama. 1999;281(9):830-4.

13. Bhatti SF, De Risio L, Munana K, Penderis J, Stein VM, Tipold A, Berendt M, Farquhar RG, Fischer A, Long S, et al. International Veterinary Epilepsy Task Force consensus proposal: medical treatment of canine epilepsy in Europe. BMC Vet Res. 2015;11:176.

14. Podell M, Volk HA, Berendt M, Loscher W, Munana K, Patterson EE, Platt SR. 2015 ACVIM Small Animal Consensus Statement on Seizure Management in Dogs. J Vet Intern Med. 2016;30(2):477-90.

15. Charalambous M, Brodbelt D, Volk HA. Treatment in canine epilepsy-a systematic review. BMC Vet Res. 2014;10:257.

16. Guyatt GH, Oxman AD, Vist GE, Kunz R, Falck-Ytter Y, Schunemann H, Grp GW. GRADE: what is "quality of evidence" and why is it important to clinicians? Brit Med J. 2008;336(7651):995-999b.

17. Holmes MA. Philosophical foundations of evidence-based medicine for veterinary clinicians. Javma-J Am Vet Med A. 2009;235(9):1035-9.

18. Higgins J. A., C. SJ: In: Higgins JPT, Green S, eds. Cochrane Handbook for Systematic Reviews of Interventions Version 5.1.0. Part 2: General methods for Cochrane reviews, Chapter 8: Assessing risk of bias in included studies, Table 8.5.d: Criteria for judging risk of bias in the 'Risk of bias' assessment tool. In: The Cochrane Collaboration. edn.; 2011. 
19. Hooijmans CR, Rovers MM, de Vries RB, Leenaars M, Ritskes-Hoitinga M, Langendam MW. SYRCLE's risk of bias tool for animal studies. BMC Med Res Methodol. 2014;14:43.

20. Engel O, von Klopmann T, Maiolini A, Freundt-Revilla J, Tipold A. Imepitoin is well tolerated in healthy and epileptic cats. BMC Vet Res. 2017;13(172):1-7.

21. Boothe DM, George KL, Couch P. Disposition and clinical use of bromide in cats. J Am Vet Med Assoc. 2002;221(8):1131-5.

22. Baho MJ, Hostutler R, Fenner W, Corn S. Suspected phenobarbital-induced pseudolymphoma in a cat. J Am Vet Med Assoc. 2011;238(3):353-5.

23. Ducote JM, Coates JR, Dewey CW, Kennis RA. Suspected hypersensitivity to phenobarbital in a cat. J Feline Med Surg. 1999;1(2):123-6.

24. Lieser J, Schwedes CS. Pseudolymphoma in a cat on phenobarbital treatment. J Small Anim Pract. 2017

25. Finnerty KE, Barnes Heller HL, Mercier MN, Giovanella CJ, Lau WW, Rylander $\mathrm{H}$. Evaluation of therapeutic phenobarbital concentrations and application of a classification system for seizures in cats: 30 cases (2004-2013). J Am Vet Med Assoc. 2014:244(2):195-9.

26. Lowrie M, Thomson S, Bessant C, Sparkes A, Harvey RJ, Garosi L. Levetiracetam in the management of feline audiogenic reflex seizures: a randomised, controlled, open-label study. J Feline Med Surg. 2017;19(2):200-6.

27. Pakozdy A, Sarchahi AA, Leschnik M, Tichy AG, Halasz P, Thalhammer JG. Treatment and long-term follow-up of cats with suspected primary epilepsy. J Feline Med Surg. 2012;15(4):267-73.

28. Volk H, Coleing JAL, Platt S, Chandler K. Clinical presentation and response to treatment of cats with epilepsy. Birmingham: BSAVA congress; 2007.

29. Boydell P. Rub epilepsy in a cat. Vet Rec. 2010;166(10):308.

30. Cuff DE, Bush WW, Stecker MM, Williams DC. Use of continuous electroencephalography for diagnosis and monitoring of treatment of nonconvulsive status epilepticus in a cat. J Am Vet Med Assoc. 2014;244(6):708-14.

31. Wahle AM, Bruhschwein A, Matiasek K, Putschbach K, Wagner E, Mueller RS, Fischer A. Clinical characterization of epilepsy of unknown cause in cats. J Vet Intern Med. 2014;28(1):182-8.

32. Cochrane SM, Black WD, Parent JM, Allen DG, Lumsden JH. Pharmacokinetics of phenobarbital in the cat following intravenous and oral administration. Can J Vet Res. 1990:54(1):132-8.

33. Cochrane SM, Parent JM, Black WD, Allen DG, Lumsden JH. Pharmacokinetics of phenobarbital in the cat following multiple oral administration. Can J Vet Res. 1990;54(3):309-12.

34. Solomon GE, Hilgartner MW, Kutt H. Phenobarbital-induced coagulation defects in cats. Neurology. 1974;24(10):920-4.

35. Gasper JAD, Heller HLB, Robertson M, Trepanier LA. Therapeutic serum phenobarbital concentrations obtained using chronic transdermal administration of phenobarbital in healthy cats. Journal of Feline Medicine and Surgery. 2015;17(4):359-63.

36. Volk HA, Chandler KE, Cappello R, Cherubini GB: New insights into efficacy and side effects of potassium bromide in epileptic cats. In: 24th American College of Veterinary Internal Medicine: 2006; Louisville, KY: Journal of Veterinary Internal Medicine; 2006.

37. Bertolani C, Hernandez J, Gomes E, Cauzinille L, Poujade A, Gabriel A. Bromide-associated lower airway disease: a retrospective study of seven cats. J Feline Med Surg. 2012;14(8):591-7.

38. Wagner S. Lower airway disease in cats on bromide therapy for seizures. In: 19th American College of Veterinary Internal Medicine: 2001. Denver, CO: Journal of Veterinary Internal Medicine; 2001

39. Klang A, Schmidt P, Pakozdy A, KneissI S, Schwendenwein I, Hirt R. Histopathological pulmonary changes in a cat with potassium bromideinduced lower airway disease. Wiener Tierärztliche Monatsschrift - Veterinary Medicine Austria. 2012:99:34-7.

40. Bailey KS, Dewey CW, Boothe DM, Barone G, Kortz GD. Levetiracetam as an adjunct to phenobarbital treatment in cats with suspected idiopathic epilepsy. J Am Vet Med Assoc. 2008;232(6):867-72.

41. Dewey CW, Barone G, Boothe DM, Smith K, O'Connor JH. The use of oral levetiracetam as an add-on anticonulsant drug in cats receiving phenobarbital. In: ACVIM forum, vol. 19. Baltimore, MD: Journal of Veterinary Internal Medicine; 2005. p. 458

42. Carnes MB, Axlund TW, Boothe DM. Pharmacokinetics of levetiracetam after oral and intravenous administration of a single dose to clinically normal cats. Am J Vet Res. 2011;72(9):1247-52.
43. Barnard LR, Barnes Heller HL. Pharmacokinetic analysis of single dose extended release levetiracetam per os in healthy cats. In: Journal of Veterinary Internal Medicine: 2017. Maryland: National Harbor; 2017. p. 1225-361.

44. Schwartz-Porsche D, Kaiser E. Feline epilepsy. Probl Vet Med. 1989;1(4):628-49.

45. Center SA, Elston TH, Rowland PH, Rosen DK, Reitz BL, Brunt JE, Rodan I, House J, Bank S, Lynch LR, et al. Fulminant hepatic failure associated with oral administration of diazepam in 11 cats. J Am Vet Med Assoc. 1996;209(3):618-25.

46. Hughes D, Moreau ER, Overall LK, Van Winkle JT. Acute heptic necrosis and liver failure associated with benzodiazepine thereapy in six cats, 1986-1995. The Journal of Veterinary Emergency and Critical Care. 1996;6(1):13-120.

47. Sawchuk AS, Parker JA, Neff-Davis C, Davis EL. Primidone in the cat. Journal of the American Animal Hospital Association. 1985;21:647-50.

48. Roye DB, Serrano EE, Hammer RH, Wilder BJ. Plasma kinetics of diphenylhydantoin in dogs and cats. Am J Vet Res. 1973;34(7):947-50.

49. Brewer DM, Cerda-Gonzalez S, Dewey CW. Zonisamide therapy for refractory seizures in five cats with presumptive idiopathic epilepsy. In: ACVIM forum: 2010; California: Journal of Veterinary Internal Medicine; 2010. p. $660-795$

50. Hasegawa D, Kobayashi M, Kuwabara T, Ohmura T, Fujita M, Orima H. Pharmacokinetics and toxicity of zonisamide in cats. J Feline Med Surg. 2008;10(4):418-21.

51. Ukai M, Hamamoto $Y, Y u$ Y, Fujiwara-Igarashi A, Fujita M, Hasegawa D. Efficacy of zonisamide on interictal electroencephalography in familial spontaneous epileptic cats. Journal of Feline Medicine and Surgery. 2017;

52. Cautela MA, Dewey CW, Schwark WS, Cerda-Gonzalez S, Badgley BL. Pharmacokinetics of oral pregabalin in cats after single dose administration. In: ACVIM forum: 2010. Anaheim, CA: Journal of Veterinary Internal Medicine; 2010. p. 739-40.

53. Pellegrini A, Gloor P, Sherwin AL. Effect of valproate sodium on generalized penicillin epilepsy in the cat. Epilepsia. 1978;19(4):351-60.

54. Zoran DL, Boeckh A, Boothe DM. Hyperactivity and alopecia associated with ingestion of valproic acid in a cat. J Am Vet Med Assoc 2001:218(10):1587-1589, 1580.

55. Dreimann E. Pharmakokinetik und klinische Nebenwirkungen der Antiepileptika Carbamazepin und Valproinsäure bei der Katze: Freie Universität Berlin; 1992.

56. Moher D, Liberati A, Tetzlaff J, Altman DG, Group P. Preferred reporting items for systematic reviews and meta-analyses: the PRISMA statement. BMJ. 2009:339:b2535.

57. van Gelder NM, Koyama I, Jasper HH. Taurine treatment of spontaneous chronic epilepsy in a cat. Epilepsia. 1977;18(1):45-54

58. Wada JA, Osawa T, Wake A, Corcoran ME. Effects of taurine on kindled amygdaloid seizures in rats, cats, and photosensitive baboons. Epilepsia. 1975:16(2):229-34.

59. Zaccara G, Franciotta D, Perucca E. Idiosyncratic adverse reactions to antiepileptic drugs. Epilepsia. 2007:48(7):1223-44.

60. Zaccara G, Gangemi PF, Cincotta M. Central nervous system adverse effects of new antiepileptic drugs. A meta-analysis of placebo-controlled studies. Seizure. 2008;17(5):405-21.

\section{Submit your next manuscript to BioMed Central and we will help you at every step:}

- We accept pre-submission inquiries

- Our selector tool helps you to find the most relevant journal

- We provide round the clock customer support

- Convenient online submission

- Thorough peer review

- Inclusion in PubMed and all major indexing services

- Maximum visibility for your research

Submit your manuscript at www.biomedcentral.com/submit 